\title{
GAUSSIAN ESTIMATES AND HOLOMORPHY OF SEMIGROUPS
}

\author{
EL-MAATI OUHABAZ
}

(Communicated by Palle E. T. Jorgensen)

\begin{abstract}
We show that if a selfadjoint semigroup $T$ on $L^{2}(\Omega)$ satisfies a Gaussian estimate $|T(t) f| \leq M G(b t)|f|, 0 \leq t \leq 1, f \in L^{2}(\Omega)$ (where $G=G(t)_{t \geq 0}$ is the Gaussian semigroup on $L^{2}\left(R^{N}\right)$ and $\Omega$ is an open set of $R^{N}$ ), then $T$ defines a holomorphic semigroup of angle $\frac{\pi}{2}$ on $L^{p}(\Omega)$, $1 \leq p<\infty$. We obtain by duality the same result on $C_{0}(\Omega)$. Applications to uniformly elliptic operators and Schrödinger operators are given.
\end{abstract}

\section{INTRODUCTION}

Let $\Omega$ be an open set of $R^{N}$ (with the Lebesgue measure), and consider a selfadjoint semigroup $T=T(t)_{t>0}$ on $L^{2}(\Omega)$ with generator $A$. Then $T$ is a holomorphic semigroup with angle $\frac{\pi}{2}$, i.e., $T$ can be extended holomorphically to the maximal domain $\{z, \operatorname{Re} z>0\}$ (see $\S 1$ for the precise definition).

Assume now that $T$ interpolates on $L^{p}(\Omega), 1 \leq p<\infty$; that is, there exists for each $p$, a strongly continuous semigroup $T_{p}$ on $L^{p}(\Omega)$ with $T_{2}=T$ and satisfying $T_{p}(t) f=T_{2}(t) f(t \geq 0)$ for $f \in L^{p}(\Omega) \cap L^{2}(\Omega)$. It follows from the Stein interpolation theorem that for $1<p<\infty$ the semigroup $T_{p}$ is holomorphic on $L^{p}(\Omega)$ with angle $\theta_{p} \geq \frac{\pi}{2}\left(1-\left|\frac{2}{p}-1\right|\right)$ (see Davies [6, p. 23]). However, the case $p=1$ is more delicate.

In the case where $A$ is an elliptic operator of second order (with some smoothness conditions on its coefficients), Amann [2] showed that $T_{1}$ is holomorphic on $L^{1}(\Omega)$ if $\Omega$ is bounded and smooth; his method is based on duality arguments and the result of Stewart $[21,22]$ on $C_{0}(\Omega)$. Recently, Arendt and Batty [3] extended the result to an arbitrary open set $\Omega$ under Dirichlet boundary conditions. We also note that Kato [12] showed the holomorphy on $L^{p}\left(R^{N}\right)$, $1 \leq p<\infty$, for the Schrödinger operator $A=\Delta-V$ ( $\Delta$ is the Laplacian and $V=V_{+}-V_{-}$is a potential).

The purpose of this paper is to extend all these results to more general situations. We show the holomorphy on $L^{p}(\Omega), 1 \leq p<\infty$, for elliptic operators under more general boundary conditions without regularity on their coefficients and by assuming minimal regularity on $\Omega$. More precisely, we show in an abstract setting the following result:

Received by the editors June 28, 1993 and, in revised form, August 16, 1993.

1991 Mathematics Subject Classification. Primary 47D03, 47F05. 
Assume that the semigroup $T$ has a Gaussian estimate, i.e.,

$$
|T(t) f| \leq M G(b t)|f| \quad \text { for } 0 \leq t \leq 1 \text { and all } f \in L^{2}(\Omega)
$$

where $M$ and $b$ are positive constants and $G=G(t)_{t \geq 0}$ is the Gaussian semigroup on $L^{2}\left(R^{N}\right)$. Then $T_{p}$ is holomorphic with angle $\frac{\pi}{2}$ on $L^{p}(\Omega)$ for $1 \leq p<\infty$.

Such Gaussian estimates hold for uniformly elliptic operators and Schrödinger operators (see [6, Chapter 3; 20, Theorem B.7.1]). Our result is applicable in the following framework.

Assume that $A$ is the operator associated with the following symmetric form

$$
a(u, v)=\sum_{i, j=1}^{N} \int_{\Omega} a_{i j} D_{i} u \overline{D_{j} v} d x+\int_{\Omega} V u \bar{v} d x
$$

with $a_{i j}=a_{j i} \in L^{\infty}(\Omega)$ satisfying the ellipticity condition $\sum_{i, j=1}^{N} a_{i j}(x) \xi_{i} \bar{\xi}_{j} \geq$ $c|\xi|^{2}$ a.e. $x \in \Omega$ and $0 \leq V \in L_{\text {loc }}^{1}(\Omega)$.

The domain of $a$ is given by $D(a)=W \cap\left\{u \in L^{2}(\Omega), \int_{\Omega} V|u|^{2}<\infty\right\}$, where $W$ is a closed subspace of the Sobolev space $H^{1}(\Omega)$ which contains $H_{0}^{1}(\Omega)$.

We obtain the holomorphy of $T_{p}, 1 \leq p<\infty$, in the following cases:

(1) $W=H_{0}^{1}(\Omega)$ for $\Omega$ any open set of $R^{N}$ (this corresponds to the Dirichlet boundary conditions).

(2) $W$ satisfies the two following properties:

* $u \in W$ implies $|u| \in W$.

* If $0 \leq u \leq v, v \in W$, and $u \in H^{1}(\Omega)$, then $u \in W$.

In this case we assume that $\Omega$ has the extension property (if $W=H^{1}(\Omega)$, this corresponds to the Neumann boundary conditions).

We recall that in [2], [3], and [12] it is shown that $T_{1}$ is holomorphic with some "small" angle. To be precise, it is shown that the estimate

$$
\left\|(\lambda-A)^{-1}\right\|_{\mathscr{L}\left(L^{1}(\Omega)\right)} \leq \frac{M}{|\lambda|}
$$

holds for $\lambda$ s.t. $\operatorname{Re} \lambda>0$ (here $M>0$ is a constant).

In the present paper we show that $T_{p}, 1 \leq p<\infty$, is holomorphic with angle $\frac{\pi}{2}$, that is, the estimate $(0.2)$ holds in $\mathscr{L}\left(\bar{L}^{p}(\Omega)\right)$ in each sector $\Sigma\left(\theta+\frac{\pi}{2}\right):=$ $\left\{\lambda=r e^{i \alpha} ; r>0,|\alpha|<\frac{\pi}{2}+\theta\right\}, 0 \leq \theta<\frac{\pi}{2}$. This holomorphy in the maximal domain $\{z, \operatorname{Re} z>0\}$ answers positively and in a more general situation a question in Kato's paper [12].

We also study the holomorphy on $C_{0}(\Omega)$. We show by duality that if the Laplacian on $C_{0}(\Omega)$ is a generator of a semigroup $T_{0}$, then $T_{0}$ is holomorphic ( $\Omega$ is any open set of $R^{N}$ ). This result has been shown by Lumer and Paquet $[13,14]$. Our method gives more information on the generator and that $T_{0}$ is holomorphic with angle $\frac{\pi}{2}$.

This paper is organized as follows. In $\S 1$ we recall some known material on holomorphic semigroups. In $\S 2$, we show that Gaussian estimates $(0.1)$ imply the holomorphy on $L^{p}(\Omega), 1 \leq p<\infty$. Finally, $\S 3$ is concerned with applications to elliptic operators on $L^{p}(\Omega)$ and $C_{0}(\Omega)$. 
Remark 0.1. (1) All the semigroups considered in this paper are assumed to be strongly continuous.

(2) If $E$ is a Banach space, we denote by $\mathscr{L}(E)$ the space of bounded linear operators on $E$ and by $\|\cdot\|_{E}$ the norm of $E$.

\section{Preliminaries}

In this section we recall some known results on holomorphic semigroups. Denote by $E$ a Banach space and by $A$ a generator of a semigroup $T=T(t)_{t \geq 0}$ on $E$. By $\varrho(A)$ and $\sigma(A)$ we denote respectively the resolvent set and the spectrum of $A$.

Definition 1.1. (a) The semigroup $T$ is said to be bounded holomorphic with angle $\theta \in\left(\theta, \frac{\pi}{2}\right]$ if $T$ has an extension to the sector $\Sigma(\theta):=\left\{z=r e^{i \alpha} ; r>0\right.$, $|\alpha|<\theta\}$ which satisfies the following:

(1) $T\left(z+z^{\prime}\right)=T(z) T\left(z^{\prime}\right), z, z^{\prime} \in \Sigma(\theta)$.

(2) $z \rightarrow T(z)$ is holomorphic on $\Sigma(\theta)$.

(3) $\lim _{z \rightarrow 0, z \in \Sigma(\theta)} T(z) f=f$ for each $f \in E$.

(4) For each $\theta^{\prime}<\theta$ there exists a constant $M$ (depending on $\theta$ ) s.t. $\|T(z)\|_{\mathscr{L}(E)} \leq M$ for all $z \in \Sigma\left(\theta^{\prime}\right)$.

(b) We say that $T$ is bounded holomorphic if there exists $\theta \in\left(0, \frac{\pi}{2}\right]$ s.t. $T$ is bounded holomorphic with angle $\theta$.

The following can be found in the books on semigroup theory (see $[9,10,11$, 15 , and 17]).

Theorem 1.2. The semigroup $T$ is bounded holomorphic with angle $\theta$ if and only if $\Sigma\left(\theta+\frac{\pi}{2}\right) \subset \varrho(A)$, and for each $\theta^{\prime}<\theta \quad\left(\theta^{\prime}>0\right)$ there exists a constant $M$ s.t.

$$
\left\|(\lambda-A)^{-1}\right\|_{\mathscr{L}(E)} \leq \frac{M}{|\lambda|} \text { for all } \lambda \in \Sigma\left(\theta+\frac{\pi}{2}\right) .
$$

Theorem 1.3. The semigroup $T$ is bounded holomorphic if and only if $\Sigma\left(\frac{\pi}{2}\right) \subset$ $\varrho(A)$ and

$$
\left\|(\lambda-A)^{-1}\right\|_{\mathscr{L}(E)} \leq \frac{M}{|\lambda|} \text { for all } \lambda \in \Sigma\left(\frac{\pi}{2}\right) .
$$

Theorem 1.3 can be deduced from Theorem 1.2 by showing that if $\left\|(\lambda-A)^{-1}\right\|_{\mathscr{L}(E)} \leq \frac{M}{|\lambda|}$ for all $\lambda \in \Sigma\left(\frac{\pi}{2}\right)$, then there exists some "small" angle $\theta>0$ such that $\Sigma\left(\theta+\frac{\pi}{2}\right) \subset \varrho(A)$, and the same estimate holds in $\Sigma\left(\theta+\frac{\pi}{2}\right)$.

We give now an interesting situation where the semigroup is holomorphic. Let $E$ be a Hilbert space and denote by $(\cdot, \cdot)$ its scalar product. Assume that $A$ is a selfadjoint generator of a bounded semigroup $T$ on $E$. Then it follows by the spectral theorem that $(A u, u) \leq 0$ for all $u \in D(A)$. Moreover, we have the following well-known

Proposition 1.4. The semigroup $T$ is bounded holomorphic with angle $\frac{\pi}{2}$.

\section{2. $L^{p}$ AND $C_{0}$ HOLOMORPHY}

We keep the same notation as in the introduction. $\Omega$ is an open set of $R^{N}$ with the Lebesgue measure and $T$ a selfadjoint semigroup on $L^{2}(\Omega)$ with generator $A$. We say that $T$ has a Gaussian estimate for $0 \leq t \leq 1$ if the 
estimate (0.1) holds for $0 \leq t \leq 1$ and that $T$ has a Gaussian estimate for all $t \geq 0$ if the estimate in (0.1) holds for all $t \geq 0$. We start by the following

Proposition 2.1. If $T$ has a Gaussian estimate for $0 \leq t \leq 1$, then there exists $w \geq 0$ s.t. the semigroup $e^{-w t} T(t)_{t \geq 0}$ has a Gaussian estimate for all $t \geq 0$.

Proof. By assumption $|T(t)| \leq M G(b t)$ for $0 \leq t \leq 1$. Let $t \geq 1$, and write $t=n+s$ with $0 \leq s<1$ and $n$ a natural number. Then

$$
\begin{aligned}
|T(t)| & =|T(n) T(s)|=\left|T(1)^{n} T(s)\right| \\
& \leq M^{n+1} G(n b) G(s b)=M^{n+1} G(b t) \leq M e^{w t} G(b t)
\end{aligned}
$$

with $w=\log M$. This shows the proposition.

The Gaussian semigroup $G$ is contractive on $L^{p}\left(R^{N}\right)$ for $1 \leq p \leq \infty$. This implies that if $(0.1)$ holds, then the semigroup $T$ satisfies $\|T(\bar{t}) f\|_{L^{p}(\Omega)} \leq$ $M e^{w t}\|f\|_{L^{p}(\Omega)}$ for $f \in L^{p}(\Omega) \cap L^{2}(\Omega), 1 \leq p \leq \infty$. By the Riesz-Thorin interpolation theorem, there exists $T_{p}(t) \in \mathscr{L}\left(L^{p}(\Omega)\right)$ s.t. $T_{p}(t) f=T_{2}(t) f:=$ $T(t) f$ for $f \in L^{p}(\Omega) \cap L^{2}(\Omega), 1 \leq p \leq \infty$. One can see easily that for $1<p<\infty, T_{p}=T_{p}(t)_{t \geq 0}$ is a strongly continuous semigroup on $L^{p}(\Omega)$. Moreover, we have

Proposition 2.2. $T_{1}$ is a strongly continuous on $L^{1}(\Omega)$.

By the density argument we see that $T_{1}(t+s)=T_{1}(t) T_{1}(s)$. We do not give the proof of the strong continuity since it is contained in Theorem 2.4 .

It is easy to see from $(0.1)$ that $T(t)$ maps $L^{2}(\Omega)$ into $L^{\infty}(\Omega)$ for each $t>0$. This implies in particular that $T(t)$ is given by a kernel $K(t, x, y)$, i.e.,

$$
T(t) f(x)=\int_{\Omega} K(t, x, y) f(x) d y \quad \text { for each } t>0, f \in L^{2}(\Omega), \text { and } x \in \Omega .
$$

The same is clearly true for $T(z)$ for $z \in \Sigma\left(\frac{\pi}{2}\right)$. Denote by $K(z, x, y)$ the corresponding kernel of $T(z)$. We have

Proposition 2.3 (see [6, p. 103]). Assume that $T$ has a Gaussian estimate for all $t \geq 0$. Then there exist two positive constants $M$ and $b$ such that

$$
|K(z, x, y)| \leq \frac{M}{\left((\operatorname{Re} z)^{N / 2}\right.} \exp \left\{-\operatorname{Re} \frac{b(x-y)^{2}}{z}\right\}
$$

for $z \in \Sigma\left(\frac{\pi}{2}\right)$ and $x, y \in \Omega$.

We state now our result on the holomorphy on $L^{p}(\Omega)$. With the same notation as above, we have

Theorem 2.4. If $T$ has a Gaussian estimate for $0 \leq t \leq 1$, then there exists $w \geq 0$ s.t. the semigroup $e^{-w t} T_{p}(t)_{t \geq 0}$ is bounded holomorphic with angle $\frac{\pi}{2}$ on $L^{p}(\Omega)$ for $1 \leq p<\infty$.

Proof. From Proposition 2.1 there exists $w \geq 0$ s.t. the semigroup $e^{-w \cdot} T$ has a Gaussian estimate for all $t \geq 0$. We can then assume that $T$ has a Gaussian estimate for all $t \geq 0$ in order to work with $T$ instead of $e^{-w^{*}} T$.

We let $K_{0}(z, x, y)=\exp \left\{-\operatorname{Re} \frac{b(x-y)^{2}}{z}\right\}, \operatorname{Re} z>0$, and $k_{0}(z)$, be the function given by $k_{0}(z)(x)=\exp \left\{-\operatorname{Re} \frac{b x^{2}}{z}\right\}, x \in R^{N}$. 
Let $f \in L^{1}(\Omega) \cap L^{2}(\Omega)$. By Proposition 2.3 we have

$$
\begin{aligned}
|T(z) f(x)| & \leq M\left((\operatorname{Re} z)^{-N / 2} \int_{\Omega} K_{0}(z, x, y)|f(y)| d y\right. \\
& \leq M(\operatorname{Re} z)^{-N / 2} \int_{R^{N}} K_{0}(z, x, y)\left|f^{\sim}(y)\right| d y
\end{aligned}
$$

where $f^{\sim}(y)=f(y)$ if $y \in \Omega$ and 0 if not.

By Young's inequality we get

$$
\begin{array}{r}
\|T(z) f\|_{L^{p}(\Omega)} \leq M(\operatorname{Re} z)^{-N / 2}\left\|k_{0}(z)\right\|_{L^{1}\left(R^{N}\right)}\|f\|_{L^{p}(\Omega)} \\
\text { for } 1 \leq p<\infty \text { and } \operatorname{Re} z>0 .
\end{array}
$$

This implies that $T(z)$ can be extended to a bounded operator $T_{p}(z) \in$ $\mathscr{L}\left(L^{p}(\Omega)\right), 1 \leq p<\infty$. By density we have $T_{p}\left(z+z^{\prime}\right)=T_{p}(z) T_{p}\left(z^{\prime}\right)$, $z, z^{\prime} \in \Sigma\left(\frac{\pi}{2}\right)$.

Let $\theta \in\left(0, \frac{\pi}{2}\right)$ be fixed. The inequality (2.1) gives

$$
\begin{aligned}
\left\|T_{p}(z)\right\|_{\mathscr{L}\left(L^{p}(\Omega)\right)} & \leq M\left((\operatorname{Re} z)^{N / 2} \int_{R^{N}} \exp \left\{-\left(\frac{\sqrt{b \operatorname{Re} z} x}{|z|}\right)^{2}\right\} d x\right. \\
& \leq M\left(\frac{1}{\sqrt{b}}\right)^{N}\left(\frac{|z|}{\operatorname{Re} z}\right)^{N} \int_{R^{N}} e^{-x^{2}} d x .
\end{aligned}
$$

It follows that there exists a constant $M_{0}>0$ s.t.

$$
\left\|T_{p}(z)\right\|_{\mathscr{L}(L p(\Omega))} \leq M_{0}\left(\frac{1}{\cos \theta}\right)^{N} \text { for } z \in \Sigma(\theta) \text { and } 1 \leq p<\infty .
$$

Consequently, $\left\|T_{p}(z)\right\|_{\mathscr{L}\left(L^{p}(\Omega)\right)}$ is bounded in $\Sigma(\theta)$.

We show that $z \rightarrow T_{p}(z)$ is holomorphic from $\Sigma(\theta)$ in $\mathscr{L}\left(L^{p}(\Omega)\right)$. It is known that this is equivalent to the weak holomorphy, i.e., $z \rightarrow\left\langle T_{p}(z) f, g\right\rangle$ holomorphic for each $f \in L^{p}(\Omega)$ and $g \in L^{q}(\Omega)$ with $\frac{1}{p}+\frac{1}{q}=1$, and $\langle\cdot, \cdot\rangle$ is the pairing between $L^{p}(\Omega)$ and $L^{q}(\Omega)$. We treat the important case $p=1$ (for $p \neq 1$ the proof is exactly the same).

Let $f \in L^{1}(\Omega)$ and $g \in L^{\infty}(\Omega)$. Consider a sequence $\left(f_{n}\right)_{n} \in L^{1}(\Omega) \cap L^{2}(\Omega)$ converging to $f$ in $L^{1}(\Omega)$. Let $g_{n}=\chi_{\Omega_{n}} g$ where $\Omega_{n}$ is a sequence of bounded open sets s.t. $\bigcup_{n} \Omega_{n}=\Omega$ and $\chi_{\Omega_{n}}$ is the indicator function of $\Omega_{n}$.

For each $n \geq 0,\left\langle T_{1}(z) f_{n}, g_{n}\right\rangle=\left\langle T(z) f_{n}, g_{n}\right\rangle$, so $z \rightarrow\left\langle T_{1}(z) f_{n}, g_{n}\right\rangle$ is holomorphic since $f_{n}, g_{n} \in L^{2}(\Omega)$. But inequality (2.2) implies that

$$
\left|\left\langle T_{1}(z) f_{n}, g_{n}\right\rangle\right| \leq M_{0}\left(\frac{1}{\cos \theta}\right)^{N}\left\|f_{n}\right\|_{L^{1}(\Omega)}\left\|g_{n}\right\|_{L^{\infty}(\Omega)} .
$$

Hence, there exists a constant $M_{1}>0$ s.t.

$$
\left|\left\langle T_{1}(z) f_{n}, g_{n}\right\rangle\right| \leq M_{1}\left(\frac{1}{\cos \theta}\right)^{N}\|f\|_{L^{1}(\Omega)}\|g\|_{L^{\infty}(\Omega)}
$$

$$
\text { for all } n \geq 0 \text { and } z \in \Sigma(\theta) \text {. }
$$

On the other hand $\left\langle T_{1}(z) f_{n}, g_{n}\right\rangle \rightarrow\left\langle T_{1}(z) f, g\right\rangle$ when $n \rightarrow \infty$. It follows from (2.3) and Vitali's theorem (see [10, Theorem 3.14.1]) that $z \rightarrow\left\langle T_{1}(z) f, g\right\rangle$ is holomorphic in $\Sigma(\theta)$. 
We show now that $T_{1}(z) f \rightarrow f$ when $z \rightarrow 0, z \in \Sigma(\theta)$ for each $f \in L^{1}(\Omega)$. Because of (2.2) it suffices to show this for $f \in L^{1}(\Omega) \cap L^{2}(\Omega)$. In this case $T_{1}(t) f=T(t) f$ for $t \geq 0$ and $T(t) f \rightarrow f$ in $L^{2}(\Omega)(t \downarrow 0)$. Consequently, we can extract a sequence $t_{n}>0$ s.t. $T_{1}\left(t_{n}\right) f(x) \rightarrow f(x)$ for a.e. $x \in \Omega$ $\left(t_{n} \downarrow 0\right)$. By the Gaussian estimate and the dominated convergence theorem we have $T_{1}\left(t_{n}\right) f \rightarrow f$ in $L^{1}(\Omega)$. The holomorphy of $z \rightarrow T_{1}(z)$ implies that $T_{1}(z) f \rightarrow f$ in $L^{1}(\Omega)$ when $z \rightarrow 0, z \in \Sigma(\theta)$. We have shown that $T_{1}$ is bounded holomorphic with angle $\theta$ for all $\theta \in\left(0, \frac{\pi}{2}\right)$. This implies that $T_{1}$ is bounded holomorphic with angle $\frac{\pi}{2}$. The proof is complete.

Our next result can be used in particular for Feller semigroups. Denote by $C_{0}(\Omega)$ the space of continuous functions in $\Omega$ which vanish at infinity. Assume that $T$ has a Gaussian estimate for $0 \leq t \leq 1$ and there exists a semigroup $T_{0}$ on $C_{0}(\Omega)$ s.t. $T_{0}(t) f=T(t) f$ for $f \in C_{0}(\Omega) \cap L^{2}(\Omega)$. We have

Corollary 2.5. There exists $w \geq 0$ such that the semigroup $e^{-w \cdot} T_{0}$ is bounded holomorphic with angle $\frac{\pi}{2}$ on $C_{0}(\Omega)$.

Proof. As in Theorem 2.4 we can assume that $T$ has a Gaussian estimate for all $t \geq 0$. Denote by $A_{1}$ and $A_{0}$ the generators of $T_{1}$ and $T_{0}$, and let $A_{\infty}=A_{1}^{*}$ on $L^{\infty}(\Omega)$.

We first show that $\Sigma(\pi) \subset \varrho\left(A_{0}\right)$. Since $T_{1}$ is holomorphic with angle $\frac{\pi}{2}$, then $\Sigma(\pi) \subset \varrho\left(A_{1}\right)=\varrho\left(A_{\infty}\right)$. We claim that $\varrho\left(A_{\infty}\right) \subset \varrho\left(A_{0}\right)$. In fact, let $\lambda \in \partial \sigma\left(A_{0}\right)$ (the boundary of $\left.\sigma\left(A_{0}\right)\right)$. It is known that this implies that $\lambda$ is in the approximate spectrum of $A_{0}$, i.e., there exists a sequence $f_{n} \in D\left(A_{0}\right)$ s.t. $\left\|f_{n}\right\|_{C_{0}(\Omega)}=1$ and $\left(\lambda-A_{0}\right) f_{n} \rightarrow 0$ in $C_{0}(\Omega)$ (see, for example, [15, p. 64]). But one can see easily that $A_{\infty}$ is an extension of $A_{0}$ and then $\lambda \in \partial \sigma\left(A_{\infty}\right)=\sigma\left(A_{\infty}\right) \subset(-\infty, 0]$. Hence $\sigma\left(A_{0}\right)=\partial \sigma\left(A_{0}\right) \subset \sigma\left(A_{\infty}\right)$ and the claim is shown.

Now since $\left(\lambda-A_{0}\right)^{-1}=\left(\lambda-A_{\infty}\right)^{-1}$ on $C_{0}(\Omega)$ for $\lambda>0$ this equality holds for all $\lambda \in \Sigma(\pi)$ by analytic continuation. Let $\theta \in\left(0, \frac{\pi}{2}\right)$. Then

$$
\begin{aligned}
\left\|\left(\lambda-A_{0}\right)^{-1}\right\|_{\mathscr{L}\left(C_{0}(\Omega)\right)} & \leq\left\|\left(\lambda-A_{\infty}\right)^{-1}\right\|_{\mathscr{L}\left(L^{\infty}(\Omega)\right)} \\
& =\left\|\left(\lambda-A_{1}\right)^{-1}\right\|_{\mathscr{L}\left(L^{1}(\Omega)\right)} \leq \frac{M}{|\lambda|}
\end{aligned}
$$

for all $\lambda \in \Sigma\left(\theta+\frac{\pi}{2}\right)$ by Theorems 1.2 and 2.4. This shows the corollary.

Remark 2.6. The same conclusion as in Corollary 2.5 holds if we replace $C_{0}(\Omega)$ by $C(\bar{\Omega})$, the space of continuous functions on $\bar{\Omega}$ with $\Omega$ bounded.

\section{Applications}

3.1. Elliptic operators. Let $\Omega$ be an open set of $R^{N}$ as above, and consider on $L^{2}(\Omega)$ the symmetric form

$$
a(u, v)=\sum_{i, j=1}^{N} \int_{\Omega} a_{i j} D_{i} u \overline{D_{j} v} d x+\int_{\Omega} V u \bar{v} d x \quad \text { where } D_{i}=\frac{\partial}{\partial x_{i}} .
$$

Assume that

$$
a_{i j}=a_{j i} \in L^{\infty}(\Omega) \text { and real, } 1 \leq i, j \leq N .
$$




$$
\begin{aligned}
& \sum_{i, j=1}^{N} a_{i j} \xi_{i} \bar{\xi}_{j} \geq c|\xi|^{2} \quad \text { for a.e. } x \in \Omega \\
& \quad \text { and all } \xi=\left(\xi_{1}, \ldots, \xi_{N}\right) \in C^{N}, c>0 \text { is a constant } .
\end{aligned}
$$

Let $W$ be a closed subspace of $H^{1}(\Omega)$ containing $H_{0}^{1}(\Omega)$ (here $H^{1}(\Omega)$ and $H_{0}^{1}(\Omega)$ are the classical Sobolev spaces).

The domain $D(a)$ of $a$ is given by $D(a)=W \cap\left\{u \in L^{2}(\Omega), \int_{\Omega} V|u|^{2} d x<\right.$ $\infty\}=W \cap D(V)$. It is easy to see that the form $a$ with this domain is closed. Denote by $T$ its associated selfadjoint semigroup on $L^{2}(\Omega)$.

We shall say that $W$ is an ideal of $H^{1}(\Omega)$ if the following two properties are satisfied:

(1) $u \in W$ implies $|u| \in W$.

(2) If $0 \leq v \leq u, u \in W$, and $v \in H^{1}(\Omega)$, then $v \in W$.

An example of such a $W$ is given by $W=\left\{u \in H^{1}(\Omega), u_{\mid \Gamma}=0\right\}$ where $\Gamma$ is a closed set of the boundary of $\partial \Omega$ of $\Omega$. (Here we assume that $\Omega$ is bounded and regular.)

Keeping these notation and those of $\S 2$, we have

Theorem 3.1. Under the assumptions (3.1), (3.2), and (3.3) we have

(1) If $W=H_{0}^{1}(\Omega)$ with $\Omega$ any open set of $R^{N}$, then $T_{p}$ is bounded holomorphic with angle $\frac{\pi}{2}$ on $L^{p}(\Omega), 1 \leq p<\infty$.

(2) If $W$ is an ideal of $H^{1}(\Omega)$, we assume in addition that $\Omega$ has the extension property. Then there exists $w \geq 0$ s.t. the semigroup $e^{-w} T_{p}$ is bounded holomorphic with angle $\frac{\pi}{2}$ on $L^{p}(\Omega), 1 \leq p<\infty$.

Proof. (1) If $W=H_{0}^{1}(\Omega)$, then $T$ has a Gaussian estimate for all $t \geq 0$ (see [6, Corollary 3.2.8]). We then apply Theorem 2.4.

(2) If $W$ is an ideal of $H^{1}(\Omega)$, then the semigroup $T$ corresponding to the form $a$ with domain $D(a)=W \cap D(V)$ is dominated by the semigroup $T_{H^{1}(\Omega)}$ corresponding to the form $a$ with domain $D(a)=H^{1}(\Omega) \cap D(V)$ (see [16] or [23]). Since $T_{H^{1}(\Omega)}$ has a Gaussian estimate for $0 \leq t \leq 1$ (see [6, Theorem 3.2.9]), then so has $T$. The claim follows again from Theorem 2.4.

Another important situation where we have Gaussian estimates is the case of the Schrödinger operator $\Delta-V$ where $V=V_{+}-V_{-}$with $0 \leq V_{+} \in L_{\mathrm{loc}}^{1}\left(R^{N}\right)$ and $0 \leq V_{-}$is in the Kato class. It is an easy consequence of the Feynman-Kac formula that the semigroup generated by $\Delta-V$ has a Gaussian estimate for $0 \leq t \leq 1$. (See, for example, [1, 20,7]. In [1], it is shown that Gaussian estimates hold for $\Delta-\mu$ where $\mu=\mu_{+}-\mu_{-}$is a certain smooth measure.) We then obtain that $\Delta-V$ generates a holomorphic semigroup with angle $\frac{\pi}{2}$ on $L^{p}\left(R^{N}\right)$ for $1 \leq p<\infty$. The fact that the angle of holomorphy can be $\frac{\pi}{2}$ answers positively a question in [12].

We can also show this result without referring to the Feynman-Kac formula. In fact, it is clear that the semigroup $e^{t\left(\Delta-V_{+}\right)}$generated by $\Delta-V_{+}$is dominated by the Gaussian semigroup. It follows from our result that $e^{t\left(\Delta-V_{+}\right)}$is bounded holomorphic with angle $\frac{\pi}{2}$ on $L^{1}\left(R^{N}\right)$. Moreover if $V_{-}$is in the Kato class, this means that $V_{-}$is $\Delta$-bounded with bound 0 on $L^{1}\left(R^{N}\right)$. Then $V_{-}$is 
$\Delta-V_{+}$-bounded with bound 0 . The result follows by the well-known perturbation arguments. This perturbation method has the advantage of working for complex potentials $V$ such that $\operatorname{Im} V$ and $(\operatorname{Re} V)_{-}$are in Kato class and $(\operatorname{Re} V)_{+} \epsilon$ $L_{\text {loc }}^{1}\left(R^{N}\right)$. To be precise, the operator $\Delta-V$ is seen here as a perturbation of $\Delta-\operatorname{Re} V$ by $-i \operatorname{Im} V$.

3.2. Laplacian on $C_{0}(\Omega)$. In this subsection we give an application of Corollary 2.5 to the Laplacian $\Delta$ on $C_{0}(\Omega)$ with $\Omega$ any open set of $R^{N}$. The result has been obtained in $[13,14]$, but their method does not give that the angle of holomorphy can be $\frac{\pi}{2}$.

Proposition 3.2. Assume that there exists a realization $A_{0}$ of the Laplacian which generates a semigroup on $C_{0}(\Omega)$. Then $D\left(A_{0}\right)=\left\{u \in C_{0}(\Omega), \Delta u \in C_{0}(\Omega)\right\}$.

Proof. Let $u \in C_{0}(\Omega)$ s.t. $\Delta u \in C_{0}(\Omega)$. Let $\lambda>0 \quad\left(\lambda \in \varrho\left(A_{0}\right)\right)$. There exists $v \in D\left(A_{0}\right)$ s.t. $\lambda u-\Delta u=\lambda v-A_{0} v$. This implies that $\lambda(u-v)-\Delta(u-v)=0$ (in the distributional sense). It is known by regularity results that this implies $u-v$ is a $C^{\infty}$ function on $\Omega$ (see, e.g., [5]). The maximum principle implies that $u=v$ since $u-v \in C_{0}(\Omega)$.

Theorem 3.3. Assume that the Laplacian $\Delta$ with domain $D(\Delta)=\left\{u \in C_{0}(\Omega)\right.$, $\left.\Delta u \in C_{0}(\Omega)\right\}$ generates a semigroup $T_{0}$ on $C_{0}(\Omega)$. Then $T_{0}$ is bounded holomorphic with angle $\frac{\pi}{2}$.

Proof. Consider the following form of the Dirichlet Laplacian on $L^{2}(\Omega)$

$$
a(u, v)=\sum_{i=1}^{N} \int_{\Omega} D_{i} u \overline{D_{i} v} d x, \quad D(a)=H_{0}^{1}(\Omega) .
$$

Denote by $T_{2}$ its associated semigroup. Then $T_{2}$ has a Gaussian estimate for all $t \geq 0$ (more precisely, we have $T_{2}(t) \leq G(t)$ for $t \geq 0$; see [6, Theorem 2.1.6] or [16, Proposition 4.2]). We have to show that $T_{2}(t) f=T_{0}(t) f$ for $f \in C_{0}(\Omega) \cap L^{2}(\Omega)$ and apply Corollary 2.5 .

If $\Omega$ is bounded and sufficiently smooth, then $T_{2}(t) f=T_{0}(t) f\left(f \in C_{0}(\Omega) \cap\right.$ $L^{2}(\Omega)$ ) for all $t \geq 0$ (see, for example, [8, Theorem 8.30; 4, p. 32]).

Let now $\left(\Omega_{n}\right)_{n \geq 1}$ be a sequence of bounded and of class $C^{\infty}$ open sets s.t. $\Omega_{n} \subset \Omega_{n+1}$ and $\bigcup_{n} \Omega_{n}=\Omega$. For each $n$, define on $L^{2}(\Omega)$ the form $a_{n}$ by

$$
a_{n}(u, v)=\sum_{i=1}^{N} \int_{\Omega_{n}} D_{i} u \overline{D_{i} v} d x, \quad D(a)=H_{0}^{1}\left(\Omega_{n}\right) .
$$

Denote by $R(\lambda, a)$ and $R\left(\lambda, a_{n}\right)$ for $\lambda>0$ the resolvents of the associated operators with $a$ and $a_{n}$ respectively. The sequence $a_{n}$ satisfies $a_{n+1} \leq a_{n}$ in the sense that $D\left(a_{n}\right) \subset D\left(a_{n+1}\right)$ and $a_{n+1}(u, u) \leq a_{n}(u, u)$. It follows by a convergence theorem on forms $([11, \mathrm{p} .452]$ or $[18, \mathrm{p} .373])$ that $R\left(\lambda, a_{n}\right)$ converges strongly in $L^{2}(\Omega)$ to $R(\lambda, a)$ for all $\lambda>0$. (Here the forms $a_{n}$ are not densely defined, but the convergence theorem is still valid; see $[3, \S 7 ; 6, p$. 62]).

Define now the operators $\Delta_{n}$ on $C_{0}\left(\Omega_{n}\right)$ by

$$
D\left(\Delta_{n}\right)=\left\{u \in C_{0}\left(\Omega_{n}\right) \cap H_{0}^{1}\left(\Omega_{n}\right), \Delta u \in C_{0}\left(\Omega_{n}\right)\right\}, \quad \Delta_{n} u=\Delta u .
$$

Since $\Omega_{n}$ is bounded and regular, $R\left(\lambda, a_{n}\right)=\left(\lambda-\Delta_{n}\right)^{-1}$ on $C_{0}\left(\Omega_{n}\right) \cap L^{2}\left(\Omega_{n}\right)$ for all $\lambda>0$. 
Let now $f \in C_{0}(\Omega)$ with compact support. Then $f \in C_{0}\left(\Omega_{n}\right)$ for $n \geq n_{0}$ (for some $\left.n_{0}\right)$. Let $h_{n}=\left(\lambda-\Delta_{n}\right)^{-1} f$ and $h=(\lambda-\Delta)^{-1} f, \lambda>0$. Define $h_{n}^{\sim}$ by $h_{n}^{\sim}(x)=h_{n}(x)$ for $x \in \Omega_{n}$ and 0 if $x \in \Omega \backslash \Omega_{n}$. Then $h_{n}^{\sim} \in C_{0}(\Omega)$ and $(\lambda-\Delta)\left(h_{n}^{\tilde{L}}-h\right)=0$ on $\Omega_{n}$ with $h_{n}^{\tilde{n}}-h \in C\left(\bar{\Omega}_{n}\right)$. The maximum principle implies that $\left\|h_{n}^{\sim}-h\right\|_{C\left(\Omega_{n}\right)}=\sup _{\partial \Omega_{n}}\left|h_{n}^{\sim}-h\right|=\sup _{\partial \Omega_{n}}|h|$. But $h \in C_{0}(\Omega)$, so $\sup _{\partial \Omega_{n}}|h|$ converges to 0 when $n \rightarrow \infty$. Hence

$$
\left\|\left(\lambda-\Delta_{n}\right)^{-1} f-(\lambda-\Delta)^{-1} f\right\|_{C\left(\Omega_{n}\right)} \rightarrow 0 .
$$

In particular, $\left(\lambda-\Delta_{n}\right)^{-1} f(x) \rightarrow(\lambda-\Delta)^{-1} f(x)$ for all $x \in \Omega$. We then get that $R(\lambda, a) f=(\lambda-\Delta)^{-1} f$. This shows the theorem.

Remark 3.4. The last result is true if instead of the Laplacian we consider an elliptic operator with smooth coefficients.

\section{REFERENCES}

1. S. Albeverio, P. Blanchard, and Z. Ma, Feyman-Kac semigroups in terms of signed smooth measures, preprint BiBos n.424, Univ. Bielefeld, 1990.

2. H. Amann, Dual semigroups and second order linear elliptic boundary value problems, Israel J. Math. 45 (1983), 225-254.

3. W. Arendt and C. J. K. Batty, Absorption semigroups and Dirichlet boundary conditions, Math. Ann. 295 (1993), 427-448.

4. Th. Cazenave and A. Haraux, Introduction aux problemes d'evolution semi-lineaires, S. M. A. I., Ellipses, 1990.

5. R. Dautray and J. L. Lions, Analyse mathematiques et calcul numerique, Vol. 2, Masson, Paris, 1988.

6. E. B. Davis, Heat kernels and spectral theory, Cambridge Univ. Press, Cambridge, 1989.

7. M. Demuth and J. A. van Casteren, On spectral theory of self-adjoint Feller generators, Rev. Math. Phy. 1 (1989), 325-414.

8. D. Gilbarg and N. S. Trudinger, Elliptic partial differential equations of second order, Springer, Berlin, 1977.

9. J. A. Goldstein, Semigroups of linear operators and applications, Oxford Univ. Press, London, 1985.

10. E. Hille and R. S. Phillips, Functional analysis and semigroups, Amer. Math. Soc. Colloq. Publ., Amer. Math. Soc., Providence, RI, 1957.

11. T. Kato, Perturbation theory of linear operators, Springer-Verlag, Berlin, 1966.

12. $ـ, L^{p}$-theory of Schrödinger operators, Aspects of Positivity in Functional Analysis ( $R$. Nagel, U. Schlotterbeck, and M. Wolff, eds.), North-Holland, Amsterdam, 1986, pp. 63-78.

13. G. Lumer and L. Paquet, Semi-groupes holomorphes et equations d'evolution, C.R. Acad. Sci. Paris Sér. I. Math. 284 (1977), 237-240.

14. __ Semi-groupes holomorphes, produit tensoriel de semi-groupes et equations d'evolution, Sem. Theorie du Potential, no. 4 (1977/78) (F. Hirsch and F. Mokobodzki, eds.), Lecture Notes in Math., vol. 713, Springer-Verlag, Berlin, 1979.

15. R. Nagel (ed.), One-parameter semigroups of positive operators, Lecture Notes in Math., vol. 1184, Springer-Verlag, Berlin, 1986.

16. E. M. Ouhabaz, Invariance of closed convex sets and domination criteria for semigroups, Potential Anal. (to appear).

17. A. Pazy, Semigroups of linear operators and applications to partial differential equations, Springer-Verlag, Berlin, 1983.

18. M. Reed and B. Simon, Methods of modern mathematical physics I, Functional Analysis, revised ed., Academic Press, New York, 1980. 
19. J. P. Roth, Opérateurs dissipatifs et semi-groupes dans les espaces de fonctions continues, Ann. Inst. Fourier (Grenoble) 26 (1976), 1-97.

20. B. Simon, Schrödinger semigroups, Bull. Amer. Math. Soc. (N.S.) 7 (1982), 447-526.

21. H. B. Stewart, Generation of analytic semigroups by strongly elliptic operators, Trans. Amer. Math. Soc. 199 (1974), 141-162.

22. _ـ Generation of analytic semigroups by strongly elliptic operators under general boundary conditions, Trans. Amer. Math. Soc. 259 (1980), 299-310.

23. P. Stollmann and J. Voigt, Perturbation of Dirichlet forms by measures, preprint.

SFB 288, Technische Universität Berlin, 136, Strasse 17 Juni, 1000 Berlin 12, Germany Max-Planck Arbeitsgruppe, fB Mathematik, 10, Am Neuen Palais, 1571 Potsdam, GerMANY

Current address: Equipe d'Analyse et de Mathématiques Appliqueés, Université de Marne-laValleé 2, rue de la Butte Verte, 93166 Noisy-le-Grand, France 